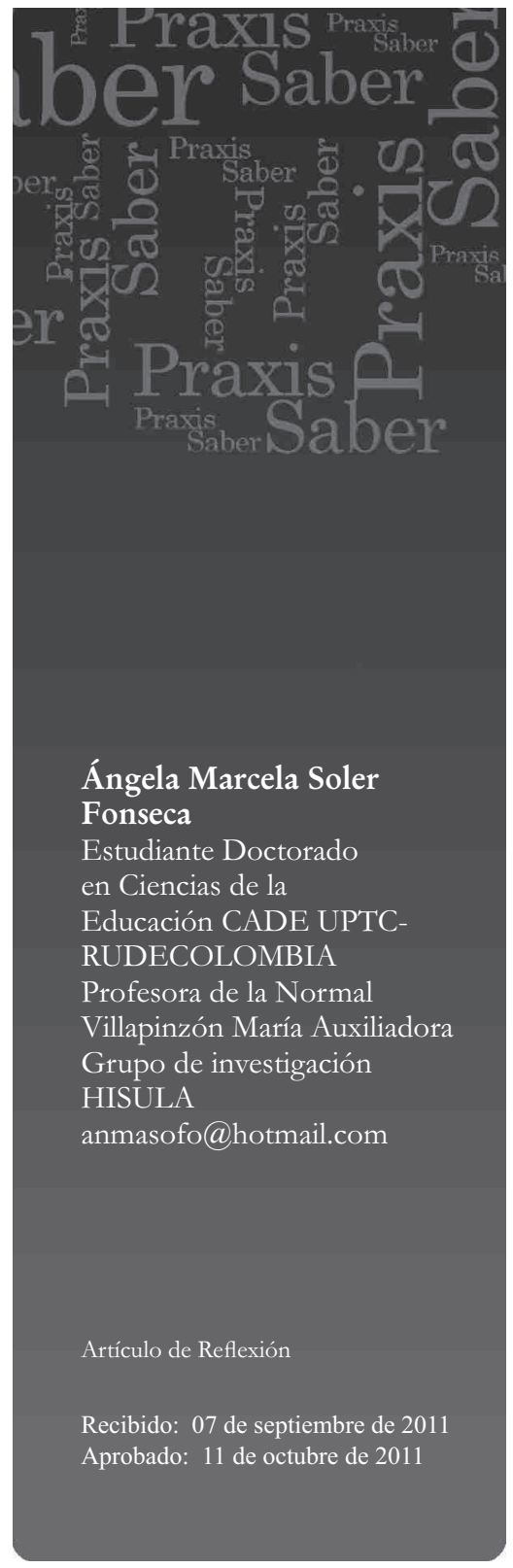

\title{
ADOLESCENCIA Y JUVENTUD EN TUNJA ${ }^{1}$
}

\section{Resumen}

Recorre las políticas sobre juventud en Colombia, y las contrasta con las realidades y acciones que se han seguido en la ciudad de Tunja en los últimos años. El Estado colombiano ha formulado políticas enfocadas a generar condiciones y ambientes propicios para los jóvenes, las cuales se han llevado a cabo con bajo impacto operativo; sin embargo, los años anteriores han sido enmarcados por la reflexión en torno al tema de adolescencia y juventud. En Tunja, para contrarrestar problemáticas de consumo de cigarrillo, alcohol, drogadicción y culturas juveniles, se han generado programas, convenios y campañas de prevención, pero en las instituciones educativas, de acuerdo con una encuesta preliminar, se evidenció el desconocimiento del mundo juvenil y de la normativa que lo respalda.

Palabras clave: Juventud, Adolescencia, Escuela, Políticas en juventud.

1 La ponencia que dio origen al presente artículo fue presentada en el II Simposio Internacional Pedagogía, Currículo y Didáctica en el contexto de la calidad de la Educación, en Barranquilla, 2010, y cuenta con reformas posteriores.

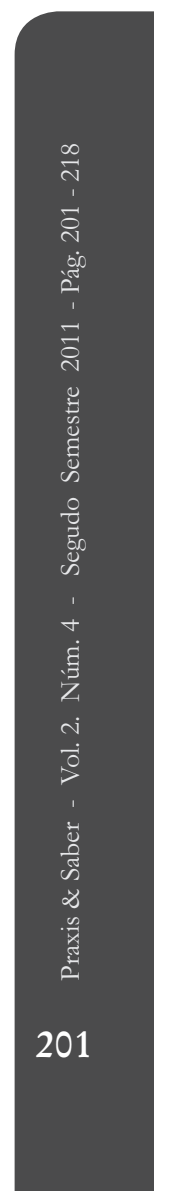




\section{Adolescence And Youths in Tunja}

\section{Summary}

This research paper examines the policies regarding young people in Colombia, and contrasts them with the actions and realities tracked in the city of Tunja over the last few years. The Colombian government has created policies focused on generating auspicious conditions and environments for young people, which have been carried out with minimal operational impact; however, the previous years have been notable for their reflection on the theme of adolescence and youths. Prevention campaigns and agreements have been created in Tunja, in order to offset problems created by the consumption of alcohol, drug addiction and youth culture; however, according to a preliminary survey in educative institutions, there is still evidence of a lack of awareness of youth culture and the laws which support it.

Key words: Youth, Adolescence, School, Youth policies

\section{Adolescence et Jeunesse À Tunja}

\section{Résumé}

On parcourt les politiques sur la jeunesse en Colombie, et les contraste avec les réalités et les actions qui ont été suivies dans la ville de Tunja au long des dernièresannées. L'état colombien a formulé des politiques qui visent la génération des conditions et des environnements propices pour les jeunes; cela a été réalisé avec un bas impact opératif. Pourtant, les dernières années ont été encadrées par la réflexion autour du sujet de l'adolescence et la jeunesse. À Tunja, on a généré des programmes, des accords et de campagnes de prévention pour contrecarrer des problématiques telles que la consommation de cigarette, d'alcool etde drogues, ainsi que les cultures des jeunes ; mais dans les institutions éducatives, selon une enquête préliminaire, il est évident la méconnaissance du monde des jeunes et de la normative qui le supporte.

Mots clés: Jeunesse, Adolescence, École, Politiques en jeunesse. 


\section{Adolescência e Juventude em Tunja}

\section{Resumo}

O texto faz um percurso pelas políticas sobre juventude na Colômbia e as contrasta com realidades e ações que se têm seguido na cidade de Tunja nos últimos anos. $\mathrm{O}$ estado Colombiano tem formulado políticas focadas para gerar condições e ambientes apropriados para os jovens, as quais se têm feito com baixo impacto operacional; porém, os anos anteriores têm sido marcados pela reflexão sobre os problemas da adolescência e da juventude. Em Tunja, para diminuir problemáticas de consumo de cigarro, álcool, drogas e práticas anti-sociais dos jovens, se tem gerado programas, convênios y multidões de prevenção, mas nas instituições educativas, baseados em uma averiguação preliminar, evidenciou-se o desconhecimento do mundo dos jovens e das normas que as regulamentam.

Palavras chave: Juventude, adolescência, escola, políticas em juventude. 


\section{Los jóvenes colombianos a través de las políticas públicas}

La juventud, entendida como fenómeno de construcción cultural ${ }^{2}$, pone en evidencia la necesidad de situarla, no solo a nivel global, sino también local, en un contexto histórico y cultural en donde sus formas de expresión y la diversidad de lo juvenil tengan significaciones pertinentes. Para abordar el tema de la juventud, en la búsqueda de su comprensión en una sociedad tan cambiante como la de hoy, es indispensable acercarse a su realidad, sus discursos, sus prácticas y, para este caso, a su contexto sociopolítico y de participación en las instituciones formales que la acogen.

Las investigaciones que buscan aportar a la comprensión de las realidades de la juventud y a resolver asuntos relacionados con ella (en los ámbitos laboral, social, cultural y demás) permiten formular reflexiones y poner en marcha de acciones que lleven a la construcción de políticas públicas, teniendo como iniciadores a actores expertos que están en capacidad de involucrarse e incidir en las dinámicas nacionales a nivel investigativo y político. Por medio de las políticas públicas de juventud se plantea y gestiona el desarrollo integral de esta población, y, por ende, se busca su articulación con la sociedad y el Estado.

En Colombia, desde la década de los sesenta del siglo pasado, la juventud ha venido mostrando interés por la participación y visibilidad en asuntos que antes solo concernían a la población adulta; mediante sus discursos y movilizaciones, amplios sectores juveniles lograron posicionarse en el país como sujetos activos y protagonistas de cambios significativos en pro de la construcción de una nación con políticas no excluyentes de ellos mismos. En los años ochenta se concretan y formalizan en Colombia los estudios sociales, que ponen en evidencia la debilidad en la comprensión de diversas problemáticas (como la violencia de nuestro país, en la que se vieron inmersos los niños y adolescentes de manera progresiva), y dan los primeros pasos para la generación de Políticas de Juventud, que le permitan a esta una participación más democrática, en ámbitos en los que antes no podía incursionar.

2 Considerada por Feixa (1995) como una condición transitoria que está marcada por la poca integración con una cultura hegemónica, lo que resta importancia a sus discursos y suma espacios de dominación por medio del poder legitimado por otros: las instituciones adultas. Por lo tanto, afirma que deben ser tenidos en cuenta como fenómeno de construcción cultural en la medida en que son captadores del futuro y modelan tendencias de cambio que contribuyen a la reinvención de la democracia y renovación social. 
A partir de 1985, que fue declarado "Año internacional de la juventud" por la Asamblea General de Naciones Unidas, el tema alcanzó importancia pública, y con la creación de la Consejería Presidencial para la Juventud, la Mujer y la Familia, en 1990, surgió la necesidad de formular la primera Política Pública Nacional de Juventud, con el ánimo de dar fuerza a los espacios de participación juvenil e integración. En 1992, con el documento CONPES 2626, la educación, el deporte y la drogadicción dejaron de ser los puntos estratégicos del trabajo con los jóvenes, y se posicionaron como criterios básicos de acción la participación, organización, descentralización y la prioridad en la atención y equidad entre géneros. Los primeros lineamientos generales para trabajar con jóvenes fueron planeados, dando un primer paso en la construcción de una nueva mirada social en el país. En 1994 se crea el Viceministerio de la Juventud, y con él, la oportunidad de tener una representación juvenil en el gobierno nacional, en los departamentos y los municipios.

En 1995 aparece una segunda Política de juventud (CONPES 2794), en donde se reconoce a la población joven como sujetos de derechos y deberes, y se plantean algunos beneficios que los integrarían a la sociedad: tarjeta joven, centros de información juveniles y ecoturismo juvenil, entre otros. Se consideró que los jóvenes deberían ser incorporados por el Gobierno para planear y ejecutar planes de desarrollo, y se canalizaron los esfuerzos en su estudio. Así mismo, se incluyeron como protagonistas en la construcción de su proyecto de vida y de la región, asumiéndolos como ciudadanos activos y plenos en derechos y deberes, por lo que surgieron los Consejos Municipales y Distritales de Juventud. En 1997 se promulgó la Ley de Juventud (Ley 375), que sigue vigente, visibilizando y motivando la participación democrática de esta población. ${ }^{3}$

En 1999 se presentaron dos hechos importantes a nivel nacional: surge el Programa de la Promoción y Protección de los Derechos de los Jóvenes, y se llevó a cabo el Seminario Taller Internacional sobre Políticas e Institucionalidad Pública de Juventud, en donde nació la estrategia "La juventud colombiana de cara al tercer milenio", que enfocó sus intereses

3 Próxima a ser derogada debido a que está en transición la aprobación de la "Ley Estatutaria de Ciudadanía Juvenil". Para mayor información véase el Proyecto de Ley Nro.169 de 2011, Senado; 014 de 2011, Cámara -acumulado con los proyectos de ley números 045 de 2011, Senado, y 084 de 2011, Cámara. Para Osorio (2012) este fue un proyecto de Ley construido y debatido por jóvenes de diferentes partes del país y que aprobó autonomía presupuestal, financiera y administrativa para el organismo de juventud. 
en "proporcionar herramientas para ampliar las oportunidades de los jóvenes como actores estratégicos del desarrollo y constructores de una sociedad más justa y equitativa" (Presidencia de la República, 2001).

En el 2000 se creó el programa presidencial para el Sistema Nacional de Juventud, "Colombia Joven” (Decreto 822 del 22 de Mayo), convirtiéndose en la base de la PNJ, y que estuvo relacionado con uno de los objetivos nacionales para construir el Estado: el de Construir equidad social. El año 2002 estuvo marcado por el desarrollo de las Mesas de Diálogo: "Presente y Futuro de los Jóvenes", donde se concretó la necesidad de que estos se apropiaran y participaran de la vida social, económica y cultural, entre otras, en aras de la construcción de la nación. En 2004 se llevó a cabo el Foro electrónico: Bases para el Plan Decenal de Juventud 2005-2015, que marcó los derroteros para la propuesta de actualización de la Política Nacional de Juventud para Colombia; este proceso fue liderado por "Colombia Joven", en unión con la Agencia de Cooperación Técnica Alemana al Desarrollo (GTZ), y contó con la participación de representantes de varios países.

En 2008, el gobierno nacional propuso suspender el programa presidencial por recorte de presupuesto, pero en un esfuerzo de seguir repensando esta población se retomó y trabajó sobre el documento CONPES, generando así una nueva base para que en el 2009 se llevara a cabo el "Diálogo Nacional para una Plataforma Nacional de Juventud". Allí se sugirió una nueva institución con objetivos ajustados a los esquemas de los jóvenes y la sociedad colombiana de hoy. Por último, la ONU, mediante resolución 64/134 del 5 febrero de 2010, declaró como Año Internacional de la Juventud, desde el 12 de agosto de 2010 hasta el mismo día del 2011, con el lema "Diálogo y comprensión mutua", demostrando así que la labor de pensar y reflexionar en y para los jóvenes es una tarea relevante, urgente e inacabada.

Con el anterior recorrido se puede hacer una aproximación a los efectos de las políticas en juventud, a las acciones y procederes que ha tenido el gobierno nacional a lo largo de los últimos años; sin embargo, también se puede evidenciar que, aunque el trabajo ha sido constante y juicioso, "es muy poco lo que realmente se había logrado en materia de juventud durante los primeros ocho años de la década del 90" (Puentes, 2002), y que por momentos parecía retroceder para tomar impulso. De esta forma, el panorama de las políticas públicas de juventud en sus albores se dilucida cuando se afirma que: 
Adolescencia y Juventud en Tunja

Las políticas de juventud florecieron silvestres, se improvisaron, no tuvieron un norte, ni metas productivas, ni bases que las sustentaran (en este sentido no fueron públicas), ni interlocutores válidos (no participaron en ellas empresarios, gremios ni organizaciones...), ni fundamento investigativo suficiente, ni pasaron por un debate abierto y trasparente... Fueron concesiones sin efecto en la vida social, en la medida que los sectores que ejecutan políticas sociales no los tomaron en serio: los viceministerios no tuvieron presupuesto, ni presencia ni poder. El Estado tomó el tema para hacer con él protagonismo, y la sociedad civil (a través de las ONG) entró en el juego, nunca lo criticaron ni lo impugnaron. El tema deslumbró a todo el mundo y la década entera de los años 90 se gastó en aprender sobre el tema, con un costo inmenso y sin resultados equivalentes: haciendo hoy un balance podemos decir que no tenemos indicadores de logro, ni existe una agenda intencionada que permita capitalizar los aprendizajes (Muñoz, 2000).

Sarmiento Anzola (2000) destaca como logros de la Política de Juventud en Colombia el reconocimiento de la diversidad y la autonomía de la población joven, la importancia del tema en las agendas nacionales, la creación de una institucionalidad en el tema de juventud y la construcción de un marco jurídico (Constitución de 1991 y Ley 375 de 1997); dentro de las dificultades, señala la asignación de recursos escasos, la dispersión e incoherencia en la legislación, la no coordinación interinstitucional, la falta de claridad en los roles institucionales y el desconocimiento de la problemática de la población objetivo, la no apropiación social de los temas, el escaso protagonismo de los propios jóvenes, además de problemas de discontinuidad en el tiempo y escasa cobertura de los programas de acción, el no seguimiento y evaluación de resultados e impacto y la no existencia de una base social o política que defienda los derechos de los jóvenes. Con todo, en 2003 se reconfirman las debilidades en la ejecución en planes de acción, al decir que "los programas específicos de juventud han sido desordenados, coyunturales y sin una perspectiva de largo plazo. $\mathrm{Y}$ adicionalmente, los recursos asignados son claramente insuficientes" (Sarmiento et. al, 2003, p. 61).

Se puede concluir que la acción del Estado enfocada a generar condiciones y ambientes propicios para los jóvenes se ha venido ajustando, pero con bajo impacto operativo; sin embargo, los años anteriores han sido enmarcados por la reflexión y el repensar de la participación real de ellos, lo cual tuvo un auge nacional y dejó una huella sin precedentes en la historia del país. 


\section{La Política Nacional de Juventud (Ley 375 del 4 de Julio de 1997²)}

La Política Nacional de Juventud (PNJ) planteó reconocer a los jóvenes como sujetos de derecho, por lo que reafirma la condición del joven como actor de construcción de desarrollo social; por lo tanto, el documento Presente y Futuro de los Jóvenes "da vigencia a sus derechos y responsabilidades; reconoce y reafirma sus identidades y afianza sus potencialidades, resultado de consensos y acuerdos entre jóvenes, Estado y sociedad" (2001, p. 3). Así mismo, busca abrir espacios de reflexión en donde la participación y el reconocimiento de los jóvenes en la sociedad formulen y ejecuten reformas a las Políticas Públicas de Juventud, que permitan la democratización y la modernización de la gestión social. De igual manera, asume que el "desarrollo integral, la participación y la vinculación a la vida social, económica, política y cultural y el ejercicio pleno y solidario a la ciudadanía" (Ley 375 de 1997) contribuyen al desarrollo integral del país; de ahí que su tratamiento requiera de un monitoreo constante no solo del Estado, sino de la comunidad en general.

En esta ley se determina que, para Colombia, joven es la persona entre los 14 y 26 años de edad, a pesar de que para la Organización Mundial para la Salud es la que se encuentra entre los 10 y 24 años $^{5}$. Cabe aclarar que no solamente se tiene en cuenta como una condición etárea ${ }^{6}$, sino que también se asume como "el cuerpo social dotado de una considerable influencia en el presente y el futuro de la sociedad y que puede asumir responsabilidades y funciones en el progreso de la comunidad colombiana". Por otro lado, el reconocimiento del mundo juvenil es abordado como "los modos de sentir, pensar y actuar de la juventud, que se expresan por medio de ideas, valores y actitudes y de su propio dinamismo interno" (Ley 375 de 1997, Artículo 4), lo cual evidencia que se ha organizado y estructurado su concepto desde lecturas que abarquen las diversas perspectivas de dicha población.

4 En la nueva "Ley Estatutaria de Ciudadanía Juvenil", un joven es considerado entre los 14 y los 28 años.

5 Aunque permite hacer delimitaciones básicas y generalizables en muchos casos. En este orden se podría iluminar con la teoría de Piaget.

6 Quienes con sus experiencias traen consigo una transformación cultural e imaginarios por parte de sus actores y las tecnologías, de manera relevante la Internet, que asume un papel primordial en este fenómeno como agente contextualizador y herramienta recurrida para obtener información. 
Así, vale la pena preguntarnos si los esfuerzos por diseñar y dar marcha a la PNJ, y sus replanteamientos basados en nuevos y constantes estudios, han logrado articulaciones reales y pertinentes en las relaciones que se celebran entre jóvenes, sociedad y Estado; como también, si estas políticas generan respuestas asertivas a las perspectivas y necesidades actuales de la población joven de nuestro país.

Como resultado del análisis de la funcionalidad de esta Ley, se reconoce que ya es el momento de hacer reformas encaminadas al fortalecimiento y adaptación de nuevas tendencias, al encontrar que:

A trece años de haber sido promulgado el marco normativo existente sobre Juventud (Ley 375 de 1997), que tiene como objeto establecer el marco institucional y orientar políticas, planes y programas por parte del Estado y la sociedad civil para la juventud, el balance no es satisfactorio. En términos de participación sólo existe menos del $50 \%$ de los Consejos Municipales y Departamentales de Juventud CMJ-CDJ, y a la fecha no se ha creado el Consejo Nacional de Juventud" (Juventudes Colombia et a., s.f.).

\section{La adolescencia en Tunja}

Antes de iniciar el camino hacia las vivencias de la temática tratada, es imperioso llegar a acuerdos sobre la conceptualización que la Ley 375 asume, en cuanto a lo que se refiere a la adolescencia y a la juventud, que, como ya se había anotado, no debe centrarse ni basarse únicamente en lo etáreo.

En primer lugar, la adolescencia hace parte del fenómeno de construcción social, que se relaciona con las transformaciones físicas y biológicas, intelectuales y cognitivas, y de identidad y personalidad. En segundo lugar, la juventud continúa como construcción social, ahora más cercana a las transformaciones psicológicas y sociales, según Brito (1996), que varían dependiendo de las condiciones históricas y sociales de la sociedad y del propio individuo; del mismo modo, se asume como el estado de la vida en que el individuo o colectivo se incorpora gradualmente en la vida adulta, y, por ende, es de integración social.

Tunja ha afrontado cambios sociales a nivel de juventud, generados por factores múltiples, como el consumismo, la globalización de la cultura, la incertidumbre económica y la búsqueda de identidad y pertenencia a grupos que los acepten y los hagan realmente partícipes, entre otros. La 
llegada a la ciudad de personas de otras partes del país y del departamento, debido al carácter de "ciudad estudiantil" , es uno de los factores relevantes en la transformación cultural de este municipio. Así aparecen y evolucionan situaciones sociales, escolares y juveniles que se presentan tanto a nivel interno como externo de las instituciones educativas y que repercuten en la cultura escolar ${ }^{8}$ y, por ende, en la sociedad; debido a que "el niño, al entrar en la adolescencia, traza una línea de demarcación para instalarse en un territorio (vedado a los mayores) donde gustos, estilos, vestimenta, lenguaje, modos de consumo y diversiones son, por esencia y por derecho, los de su edad. Esto es a lo que se ha llamado la nueva cultura juvenil" (Perinat, 2003, p. 67).

Uno de los hechos significativos, y de reconocimiento abierto, acontecidos en Tunja, referentes a las diferentes problemáticas que tocan el mundo juvenil de manera significativa en los últimos diez años, ha sido el consumo de cigarrillo y alcohol, que llevaron a la ciudad a ser una de las más nombradas, en 2001, por el Programa Nacional Rumbos, ya que presentaba un porcentaje alto de adolescentes y jóvenes que hacían parte protagonista de este informe; circunstancia que se mantuvo hasta 2009. Lo anterior tuvo serias repercusiones, tanto a nivel del orden público como personal, lo que afectó de manera significativa la familia y los entornos sociales y, por ende, escolares. Por otro parte, y no menos relevante, estudios del Hospital San Rafael señalaron que en 1997 en Tunja se registró un intento diario de suicidio, situación que llegó a ser crítica en los años 2004 y 2009, cuando aumentaron aún más los atentados a la vida propia en la población juvenil de Boyacá, según las estadísticas de la Policía (www.eltiempo.com, 2009, junio 4) ${ }^{9}$.

Por último, en cuanto a la identidad que asumen los adolescentes, un estudio realizado en el 2008 por la Policía Comunitaria de Boyacá logró identificar grupos como los punkeros, los emos, los skinhead, los ska, los raperos, los rastafary, los hippies, los metaleros, los dark y los pijos. En ese año, el patrullero de la Policía William Wilches ${ }^{10}$ hacía alusión al aumento

7 Entendida como las maneras específicas de organizarse y comunicarse los miembros pertenecientes a una institución escolar.

8 Se toman los reportes periodísticos por ser de circulación departamental, los cuales contribuyen en la generación de comprensiones de la realidad de quienes lo leen.

9 Durante los años anteriores, era quien manejaba la información oficial y confiable acerca de los fenómenos juveniles presentados en la ciudad.

10 En los estudios cualitativos casi siempre se emplean muestras pequeñas no aleatorias, ya que aplican criterios distintos para seleccionar a los participantes. Se debe tener en cuenta que el interés de la investigación cualitativa en ocasiones se centra en un caso que presenta interés intrínseco para descubrir significado o reflejar realidades múltiples, por lo que la generalización no es un objetivo de la investigación (Salamanca 
de tribus urbanas y subculturas juveniles en Boyacá, y al auge de ellas, por moda o por presión de amigos y compañeros, más no porque existiere una ideología fuerte en ellos que impulsara la aparición de este tipo de fenómenos (www.eltiempo.com, 2008, octubre 9). Lo anterior evidencia la forma como inciden los medios de comunicación y las redes sociales en los constitutivos de la identidad, y que estos últimos ya no pasan por la escuela, puesto que las mismas normas de la institución invisibilizan y cohíben su exteriorización, pero se reproducen y fortalecen en escenarios permisivos y muy flexibles, como la calle.

Dentro de las acciones planeadas por las políticas municipales para contrarrestar estas problemáticas se encuentra, entre otras, un convenio que suscribió la Gobernación con los Padres Terciarios Capuchinos para rehabilitar aquellos jóvenes mayores de 18 años que han tenido contacto prolongado con estupefacientes (www.eltiempo.com, 2009, febrero 16), y como estrategia municipal surge, en el año 2008, una campaña de prevención para la población juvenil de la ciudad, denominada "Yo sí tomo... mi vida en serio", la cual estuvo enmarcada en el Plan de Desarrollo Municipal 2008-2011, y que surgió debido al informe de la ONG Nuevos Rumbos en el año 2006, el cual evidencia, nuevamente, el excesivo consumo de alcohol y sustancias psicoactivas en Tunja (Informe de gestión - Años 2008-2011, Secretaría de Gobierno Municipal). Este programa, inicialmente, se centró en la prevención del consumo de alcohol en menores de edad, y obtuvo el impulso de un grupo de jóvenes que visitaba los colegios y algunas universidades, brindando información sobre diversos fenómenos negativos que se posicionan con fuerza en la ciudad y que necesitan de acciones tanto individuales como grupales para ser contrarrestados.

Por último, en cuanto al impacto educativo que lo anterior genera, entran en juego problemáticas diversas, como malestar escolar por parte los miembros de las instituciones educativas, debilitamiento en el interés por las actividades académicas y, por ende, bajo rendimiento académico, el ausentismo, la falta de identidad escolar y familiar y la pérdida del valor a lo académico, social y cultural, así como al futuro vocacional y profesional. Del mismo modo, se naturalizan estos fenómenos en profesores y padres de familia frente a la realidad adolescente y juvenil, lo que impulsa a reforzar actitudes no deseables. 
En este punto cabe formular algunos interrogantes a las problemáticas anteriormente expuesta: ¿son las instituciones educativas formadoras integrales de la nueva juventud que la globalización y la demanda de consumo están generando?, ¿de qué manera se interesan las instituciones educativas en el bienestar y formación de los jóvenes que a ella llegan y articulan sus currículos con la Política Nacional de Juventud? y, por último, y quizá más importante, ¿qué ha sucedido con el esfuerzo de expertos, Estado y sociedad en el diseño y puesta en marcha de unas Políticas Nacionales de Juventud, si resalta a simple vista que muchos de los actores que participan en la educación de los adolescentes no las conocen ni asumen de manera cotidiana en nuestra ciudad?

\section{Una breve indagación}

Para conocer lo que saben y asumen los adultos respecto a las vivencias de sus estudiantes adolescentes y a las políticas nacionales de juventud, se realizó un muestreo cualitativo ${ }^{11}$ y se encuestó a un grupo de quince profesores de básica secundaria, siete coordinadores y veinte padres de familia de hijos adolescentes pertenecientes a tres instituciones educativas privadas y a tres públicas, de la ciudad de Tunja. La encuesta contó con 15 preguntas de tipo abierto, con el fin de escuchar el sentir de cada estamento, y, de esta manera, hacer un prediagnóstico de la situación juvenil. Algunos indicios arrojados en el análisis de las respuestas son:

Para la mayoría de los docentes y padres de familia, ser joven se enfoca más a la vitalidad y al dinamismo con el que cuentan algunos seres humanos; los coordinadores lo relacionan con la edad y la pertenencia a grupos que se relacionan por amistad y que en cierta forma excluyen al adulto. La juventud fue definida por la mayoría como la etapa de transición entre la niñez y la adultez, haciendo alusión a los sueños, la amistad, el proceso de madurez y el desarrollo de la libre personalidad como asuntos claves de esta etapa. Términos como irreverencia, desobediencia, desorientación, escases de valores, desinterés, rebeldía, falta de criterio e incertidumbre marcaron especialmente las respuestas más recurrentes en las encuestas realizadas en los colegios públicos, relacionándose así con el estrato socioeconómico y las oportunidades culturales de esa población. En cuanto al mundo juvenil, el consenso remite al entorno donde se

11 Que para Sierra y Rojas es una "agrupación conformada por jóvenes que se articulan en torno a la defensa de intereses comunes, con el fin de generar un ambiente propicio para compartir experiencias y rituales, configurando una visión émica del mundo" (2005, p. 143). 
desenvuelven e interactúan los jóvenes con sus pares, donde se desarrolla cierta identidad, autonomía y donde enfrentan sus realidades.

Según los encuestados, la juventud tiene varias problemáticas, especialmente en la etapa de la adolescencia, como la soledad familiar a la que se enfrentan, la permisividad actual de muchos hogares, la influencia de los medios masivos de comunicación y, en especial, la Internet y la influencia de los amigos, entre otras. Como aspecto clave, las exigencias de sus pares para pertenecer a los grupos ya conformados son derroteros que marcan el conflicto.

La mayoría advierte la incertidumbre de futuro, el vacío de proyectos de vida y el poco acatamiento de la autoridad de sus mayores y las normas. Pocos reconocen en esta población a líderes activos, participativos y asertivos que propicien cambios significativos en sus entornos, y sí los asocian con el consumismo y el libertinaje, lo que permite pensar que "en los institutos se estaría formando una cultura adolescente en la que participarían todos sin distinción de clase -con un sistema autónomo de normas y valores, esencialmente hedonista-" (Criado, 1998, p. 27).

Los encuestados conocen superficialmente sobre las tribus urbanas ${ }^{12}$ y las subculturas juveniles ${ }^{13}$, para ellos no hay diferencia entre las primeras y las segundas; conocen de estas por los medios de comunicación y por conferencias a las que han asistido de manera voluntaria. Los grupos juveniles parecen tener connotaciones negativas, especialmente para los docentes, quienes los confunden con tribus; solo unos pocos identifican la diferencia entre ellos. La mayoría reconoce las características de los emos e identifican sus comportamientos y consecuencias tanto individuales como grupales, pero de las demás no hicieron referencia; sin embargo, reconocen que existen otras y asumen, por la información que recogen de otros, que es una condición de su grupo hacerse daño entre ellos.

Así mismo, se evidencia que hay mayor influencia de las tribus urbanas en los colegios públicos, en donde invisiblemente se hacen trabajos de reclutamiento, pero que fuera de los planteles se ponen en evidencia; es en estas instituciones donde los docentes expresan mayor debilidad en cuanto a la capacitación en este tema; aun así, hay una conciencia de la existencia

12 Que para Antonio Pérez Islas (1998) es la expresión innovadora de los jóvenes para diferenciarse cada vez más de una cultura dominante y hegemónica.

13 Agrupaciones de jóvenes con fines comunes: música, arte, deporte, inquietudes, entre otras.

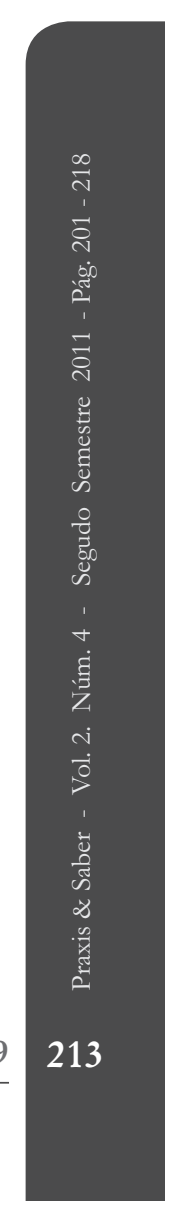


de estas tribus, y se especula sobre sus actividades. Uno de los tres colegios privados que fueron encuestados mantiene en capacitación constante a los miembros de su comunidad en este tema; por lo que quienes integraron la muestra aseguraron que no hay casos visibles de pertenencia a alguna de las tribus. Los demás no han explorado este terreno en el interior de la institución, por creer que no enfrentan dicha "problemática". Además, desconocen si sus estudiantes consumen licor, cigarrillo o drogas.

El informe de la investigación Atlántida, citado por Erazo, muestra "cómo la cultura escolar se ha aislado significativamente de la cultura social y que frente al dinamismo del cambio social, la escuela ha permanecido relativamente estática e inmodificable" (2004, p. 6). Las instituciones educativas tunjanas deben dejarse permear por lo urbano; iniciar a pensar en la investigación de sus calles para comprender cómo influyen en la escuela. Con lo anterior, para la mayoría de los encuestados los jóvenes cambian sus actitudes, vocabulario, vestuario, entre otras, cuando salen de la institución educativa, por lo que es fuera de esta donde en realidad se pueden caracterizar.

El manual de convivencia, que en muchos casos es más un reglamento estudiantil del colegio, y la autoridad unidireccional de muchos maestros inhiben la subjetividad del adolescente que desea mostrar su pertenencia a un grupo, lo que sucede en menor medida en el interior de las familias, con sus padres como figura de autoridad, puesto que en la mayoría de casos los adultos no demuestran tendencias empatizadoras con estos fenómenos juveniles y ejercen el poder para contrarrestar sus simbologías y marcas. Es necesario reconocer que "no son las relaciones entre edades las que explican el cambio de una sociedad, sino el cambio en las sociedades el que explica las relaciones entre las diferentes edades" (Allen, 1968, citado por Criado, 1998).

Las relaciones de los coordinadores con los estudiantes son pacíficas y normales, pues los primeros representan la autoridad. Varios encuestados afirman que la edad, especialmente marcada por la utilización de la tecnología, juega un papel importante dentro de los procesos de convivencia y socialización. Las brechas generacionales son notorias en los planteles educativos, encontrándose el irrespeto (burlas, contestaciones, indiferencia) a los mayores, y viceversa, como una forma de violencia escolar.

Una preocupación hallada es que la mayoría de los docentes y padres de familia encuestados no conocen la Política Nacional de Juventud, ni la Ley 1098 o de Infancia y Adolescencia. Los coordinadores, por su labor 
mediadora, han tenido capacitación, pero reconocen no ser expertos en el tema. Por otro lado, el Consejo Municipal de Juventud es comprendido por los docentes como el ente que propicia algunos espacios de opinión y lúdicos a los jóvenes; aun así, los planteles educativos han participado en las elecciones, más no en actividades significativas como para tenerlas en cuenta. Para coordinadores, maestros y padres no hay claridad sobre sus funciones y accionar. La mayoría de padres de familia aseguraron no saber que existía. Para motivar y hacer partícipe a la juventud de Tunja en los asuntos sociales, económicos, culturales, etc., es necesario contar con la colaboración de los adultos, con la capacitación de quienes directamente interactúan con ellos: docentes y padres de familia.

En cuanto a los soportes legales de los Manuales de convivencia, los docentes y padres de familia asumen que tienen como cimiento la Ley 375 y la Política de Juventud, pero no hay seguridad; la mitad de los coordinadores encuestados no están seguros o no saben, pues cuando tomaron el cargo ya estaban elaborados, y las reformas hechas han sido leves y especialmente de forma. Muy pocos reconocieron la participación en sus reformas recientes, y afirmaron haber tenido en cuenta la Ley 1098, más no las Políticas de Juventud. Para algunos padres de familia el reglamento estudiantil es más punitivo que preventivo, se centra en la crítica del estudiante y en reconocerlo como un sujeto de deberes.

Por último, para la mayoría de docentes y coordinadores, la función de los padres de familia en el desarrollo integral de los adolescentes no es muy clara y comprometida. El trabajo y los compromisos sociales de los adultos han restado importancia al acompañamiento social, cultural y académico de sus hijos. De igual forma, poco se ocupan por conocer los intereses y necesidades de sus hijos y de los amigos que los rodean, cediendo espacios importantes a personas que no responden a sus dudas e inquietudes de manera asertiva, coherente y positiva.

\section{Conclusión}

El desconocimiento de los adultos encuestados sobre el mundo juvenil y la normativa que respalda a los jóvenes puede aumentar la brecha generacional, cultural y política, permitiendo distanciamientos que se vuelven cada vez más profundos. Del mismo modo, generar esfuerzos enmarcados en el diseño, reconocimiento y apoyo de planes aislados de programas conducentes a la prevención de riesgos psicosociales, lleva al alejamiento de acompañamiento familiar, escolar y político, situación que debería plantearse inicialmente desde las instituciones educativas.

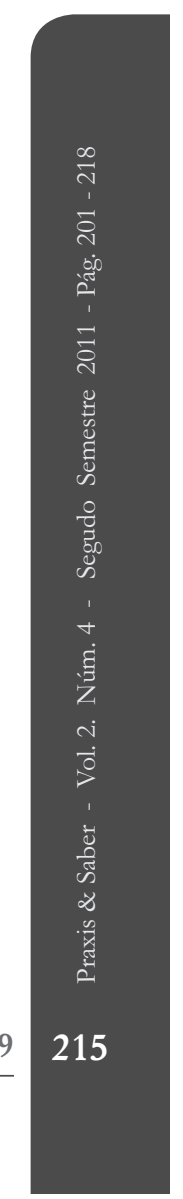


Se puede decir que el Estado no es el único encargado de la ejecución de Políticas Juveniles, y que a pesar de los esfuerzos realizados, la comunidad en general y, especialmente, la educativa tiene la responsabilidad social de apoyar la participación de los niños, los adolescentes y los jóvenes en diferentes ámbitos. Sin embargo, no se evidencia un interés significativo a nivel general, que los ubique en un lugar relevante en la sociedad, pues parecen ser los más afectados con la invisibilidad social que los aqueja, derrochando así las oportunidades de innovación y reformas planteadas por ellos como sujetos generadores de cambio, inmersos en instituciones tradicionales.

Así, las instituciones educativas, representadas por sus directivos, deben ser las principales encargadas de capacitar, en primera medida, a los docentes y padres de familia, quienes son los que tienen el contacto directo y constante con esta población, con el fin de fortalecer el conocimiento de las leyes y programas enfocados al desarrollo de las metas propuestas por estas, y lograr que adolescentes y jóvenes transiten de manera natural por lo que el Estado les brinda; además, para que sean protagonistas de sus propios cambios, proyectos y trayectorias, y para que por medio del conocimiento de la normativa sean ellos quienes busquen ser partícipes de las transformaciones significativas del entorno, que otorgue oportunidades de participación y seguridad en su región. Es por esto que la relación entre políticas juveniles y trabajo escolar debe localizar sus puntos de encuentro y concertar planes de acción que converjan y proyecten entre sí.

De igual manera, es vital comprender que los principales distanciamientos entre adolescentes, jóvenes y adultos son provocados por la escasa interacción y el desconocimiento de sus mundos en determinados momentos. La imagen del adolescente y el joven de Tunja no cuenta con bases sólidas, dado que se han seguido pensando como el desequilibrio de la sociedad, por los imaginarios tradicionales que perviven, aun cuando la sociedad ha evolucionado por la globalización y por las tendencias internacionales de interculturalidad, entre otras; ya que puede existir la tendencia de que la cultura dominante los reprima, los excluya y pormenorice sus ideales, situación que debe evolucionar por medio de la apertura de caminos de concientización, comprensión y tolerancia, por parte de la población adulta en el nivel que se encuentren.

Los adolecentes y jóvenes de Tunja deben constituirse como sujetos con potencialidades y capacidades emergentes de nuevos desafíos, y gracias a que son un potencial de oportunidades en todos los ámbitos, se pueden explorar vocaciones, discursos y experiencias, ente otros, que enriquezcan el mundo del adulto. 


\section{Referencias}

Alcaldía Mayor de Tunja (2008). Informe de gestión. Proyecto de seguridad "Yo sí tomo... mi vida en serio". Años 2008-2011. Emitido por la Secretaría de Gobierno Municipal y el Equipo Proyecto. Tunja.

Brito Lemus, Roberto (1996). "Hacia una sociología de la juventud”. Jóvenes, Revista de estudios sobre juventud, N. ${ }^{\circ}$ 1. México: IMJ.

Criado, Enrique (1998). Producir la juventud. Crítica de la sociología de la juventud. Madrid: ITSMO.

DÁvila LEÓN, Óscar (2004). "Adolescencia y juventud: de las nociones a los abordajes". Revista Última Década. 12(21). Santiago de Chile. Disponible en: http://www.scielo.cl/scielo.php?pid=S071822362004000200004\&script=sci_arttext

El Tiempo (2008, octubre 9). Aumenta presencia de Tribus Urbanas en Boyacá. Sección Nación. Disponible en http://www.eltiempo.com/ archivo/documento/CMS-4593962

El Tiempo (2009, febrero 16). Mayores consumidores de drogas en Boyacá son Tunja, Duitama, Sogamoso y Villa de Leyva. Sección Nación. Disponible en: http://www.eltiempo.com/archivo/documento/CMS4819014

El Tiempo (2009, Junio 4). AUtORIDADES ENCIENDEN ALARMAS POR AUMENTO de suicidios en BOYACÁ EN EL 2009. SeCción NaCión. Disponible en: HTTP:/ /WWW.ELTIEMPO.COM/ARCHIVO/DOCUMENTO/CMS-5356027

El Tiempo (2010, marzo 22). Gobierno departamental implementó 'Teléfono Salvavidas' para bacerle frente al suicidio en Boyacá. Sección Nación. Disponible en: http://www.eltiempo.com/archivo/documento/CMS7458589.Erazo, E. (2004). "Contexto de crisis de sentido y su relación con el desarrollo moral en la escuela colombiana". Revista de Ciencias Sociales, Niñez y Juventud, 2(2). CINDE, Centro de Estudios Avanzados en Niñez y Juventud, Manizales, Colombia.

Fexia, Carles (1995). El reloj de arena. México: Causa joven.

Manrique Abril, Fred Gustavo; Ospina Díaz, Juan Manuel y Herrera Amaya, Giomar Maritza (2009). Consumo de sustancias psicoactivas y factores determinantes en adolescentes de la ciudad de Tunja 2009. UPTC-Secretaría de Protección Social de Tunja.

Muñoz GonzÁLEz, Germán (2003). "Temas y problemas de los jóvenes colombianos al comenzar el siglo XXI". Revista Latinoamericana de Ciencias Sociales, Niñez y Juventud, 1(1). Enero-Junio 2003.

Centro Internacional de Educación y Desarrollo Humano, CINDE, Universidad de Manizales, Colombia. Disponible en: http:/ /

bibliotecavirtual.clacso.org.ar/ar/libros/colombia/cinde/revis1/06.pdf

Perinal Maceres, Adolfo (Coord.) (2003). Los adolescentes en el siglo XXI: un enfoque psicosocial. Editorial UOC. 
Puentes, Julio (2002). Políticas públicas de juventud y gasto público en Colombia (1991-2001). Tesis de grado, Magíster de Economía, Universidad Nacional. Bogotá. Disponible en: http://www.unicef.org/ colombia/pdf/PoliticasJuv3.pdf

Puentes, Julio (2003). "Finanzas públicas, niñez y juventud".

República de Colombia (1997). Ley 375. Ley de Juventud, 4 de julio de 1997.

República de Colombia, Presidencia de la República (2001). Hacia una política pública de juventud en Colombia. Herramientas para su construcción e institucionalización. Extraído el 1 de mayo de 2010 de http://juventudcb.com/Text/POLITICA\%20DE\%20 JUVENTUD $\% 20$ EN $\% 20$ COLOMBIA.pdf

Salamanca Castro, Ana Belén y Crespo Blanco, Cristina Martín (2007). "El muestreo en la investigación cualitativa". Revista Nure Investigación, n. ${ }^{\circ}$ 27, marzo-abril, 2007. Disponible en: http://www.nureinvestigacion. es/FICHEROS_ADMINISTRADOR/F_METODOLOGICA/ FMetodologica_27.pdf

Sarmiento Anzola, Libardo (2000). Política pública de juventud en Colombia. Logros, dificultades y perspectivas. Disponible en: http://207.58.191.15:8180/xmlui/handle/123456789/100

Sarmiento, Alfredo; GonzÁlez, Jorge Iván; Delgado, Liliana; Martínez, Rafael y Osorio, Alejandra (2012). ¿En qué va la ley estatutaria de ciudadania juvenil? Disponible en la web: http://www.slideshare.net/ observatoriojuventud/leyestatutaria

SIERRA, L. S y RojAs M.F. (2005) Violencias contra jóvenes. Bogotá: Pontificia Universidad Javeriana. 